\title{
A comment on "Species are not most abundant in the centre of their geographic range or climatic niche"
}

\author{
Jorge Soberón', A. Townsend Peterson', Luis Osorio-Olvera² \\ I Department of Ecology and Evolutionary Biology and Biodiversity Institute, University of Kansas, Lawrence, \\ KS 66049, USA 2 Facultad de Ciencias, Universidad Nacional Autónoma de México, Cd. Universitaria, \\ 04510 Ciudad de México, México
}

Corresponding author: Jorge Soberón (jsoberon@ku.edu.)

Academic editor: L. Winder | Received 6 March 2018 | Accepted 22 July 2018 | Published 15 August 2018

Citation: Soberón J, Peterson TA, Osorio-Olvera L (2018) A comment on "Species are not most abundant in the centre of their geographic range or climatic niche". Rethinking Ecology 3: 13-18. https://doi.org/10.3897/ rethinkingecology.3.24827

\begin{abstract}
A study published recently argued against a relationship between population density and position in geographic and environmental spaces. We found a number of methodological problems underlying the analysis. We discuss the main issues and conclude that these problems hinder a robust conclusion about the original question.
\end{abstract}

\section{Keywords}

population density, niche centrality, niche distances

\section{Introduction}

The question of whether population density is related to position in geographic (Sagarin 2002) or ecological niche space (Yañez-Arenas et al. 2012, Martínez-Meyer et al. 2013) is important and still unresolved. Niche theory essentially assumes that different environmental combinations cause differences in fitness, and therefore, ceteris paribus, in population densities. If this is true, estimating niche properties will provide information about population properties. Some empirical data supports this hypothesis (Yañez-Arenas et al. 2012, Martínez-Meyer et al. 2013). However, in a recent paper 
in Ecology Letters, Dallas et al. (2017) examined the problem using a large dataset of 118,000 sampled populations of $>1400$ species of birds, mammals, and trees. Dallas et al. (2017) failed to detect consistent and significant correlations between population density and distance to the centroids of species' distributions in geographic or environmental spaces, and concluded against the generality of such distance-density relationships.

Dallas et al. (2017) focuses on an important problem, and the dimensions of their dataset are impressive. However, the authors' failure to detect significant relationships may result from methodological artifacts, rather than to non-existence of such relationships. We would like to point to several problems inherent in the analysis of Dallas et al. (2017).

\section{Results}

1) The largest dataset analyzed by Dallas et al. (2017) was eBird observations (Sullivan et al. 2009), which are collected without any sampling protocol or plan (there are alternative and better databases, like the Breeding Bird Survey, that although biased spatially is compiled by experts using established protocols). eBird has biases frequent among observational data, like more observers near cities, and more reporting where a species is rare. Therefore, confounding effects between effort and observer bias may be present, at least for the birds.

2) Dallas et al. (2017) express caution about maximum abundances falling at the periphery of sampled ranges for two of the datasets that they analyzed, but we still worry that true niche centroids will not be represented appropriately. Dallas et al. (2017) largely disregarded parts of species' distributions falling outside the regions for which they had abundance data available. We illustrate this point using the rodent Dipodomys merriami Dallas et al. (2017). Figure 1 shows the spatial minimum convex hull $(\mathrm{CH})$ for occurrences in the United States (region in gold on the map). This is considerably less extensive than the range outline for this species from IUCN (Patterson et al. 2003). The geographic centroids based on the two range outlines are markedly distinct.

A similar problem exists in environmental space. We downloaded the 2-dimensional principal components (PC) used by Dallas et al. (2017). For 1799 localities (debugged and thinned to $0.1^{\circ}$, out of 40,000 available via GBIF), we extracted the PC values for each of the points. Figure 1 shows that the range of environmental space in the full distributional area of Dipodomys merriami extends into environmental space not represented in the $\mathrm{CH}$ used by Dallas et al. (2017).

3) Dallas et al. (2017) used CHs to characterize ecological niches of species. $\mathrm{CHs}$ are sensitive to outliers (Syväranta et al. 2013), and their centroids may be quite distinct from those obtained using robust estimators (Van Aelst and Rousseeuw 2009). In Figure 2, based on the D. merriami example, we plot in environmental space the data points used by Dallas et al. (2017). We also show two methods of characterizing the 


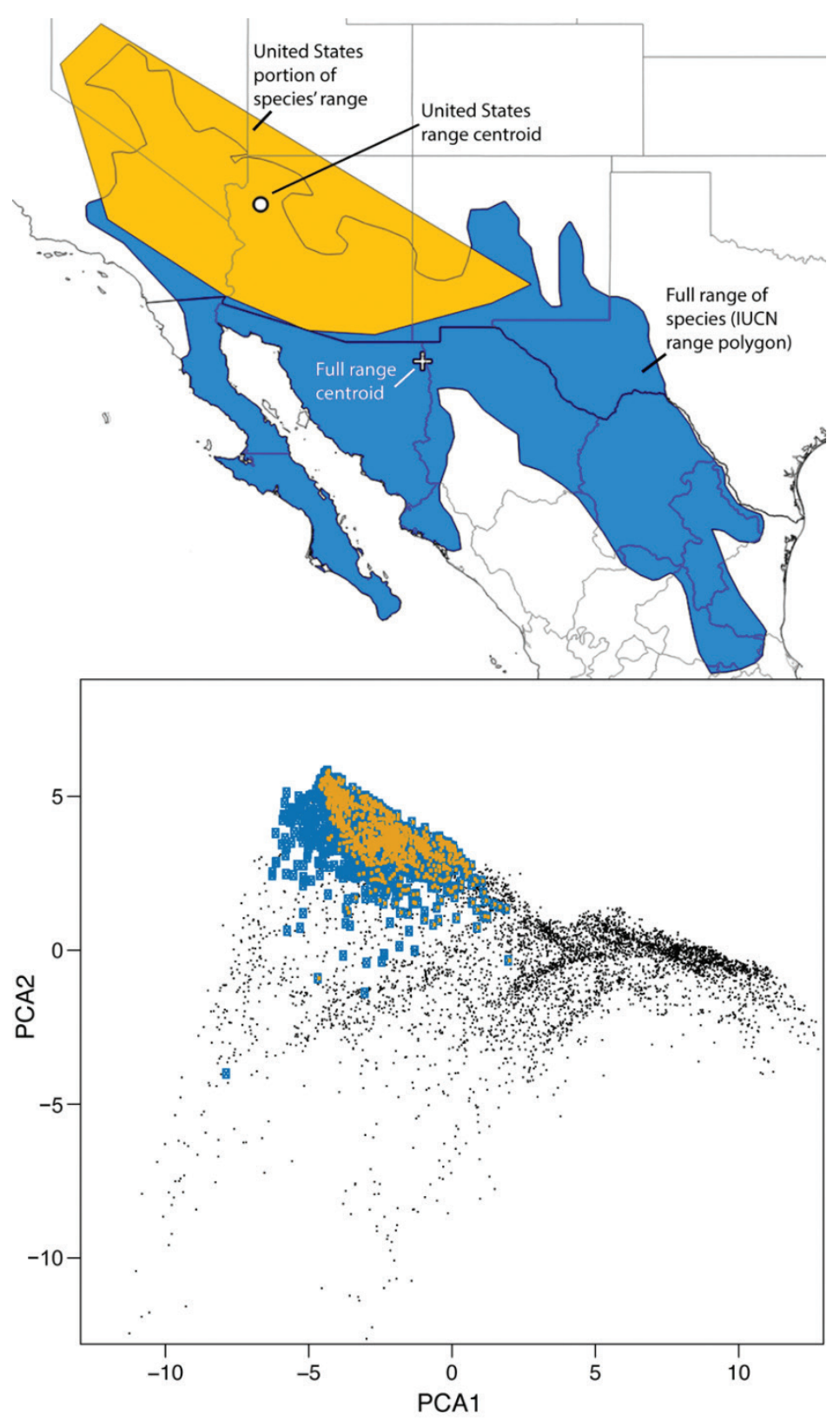

Figure I. Geographic and environmental spaces for Dipodomys merriami. Top: extent of occurrence polygon for the distribution of D. merriami in the United States (gold, centroid shown by black and white circle), representing the range area analyzed by Dallas et al. (2017), and the full range of the species (blue, centroid show as a cross; polygon from IUCN). Note that the true range centroid falls outside of the convex hull analyzed by Dallas et al. (2017). Bottom: 1799 data points from GBIF (see text) in a space of the first two climatic principal components used by Dallas. et al. (2017; see text). Points in gold are the reduced portion (United States) of the species range analyzed by Dallas et al. (2017); points in blue cover the entire range of the species. 


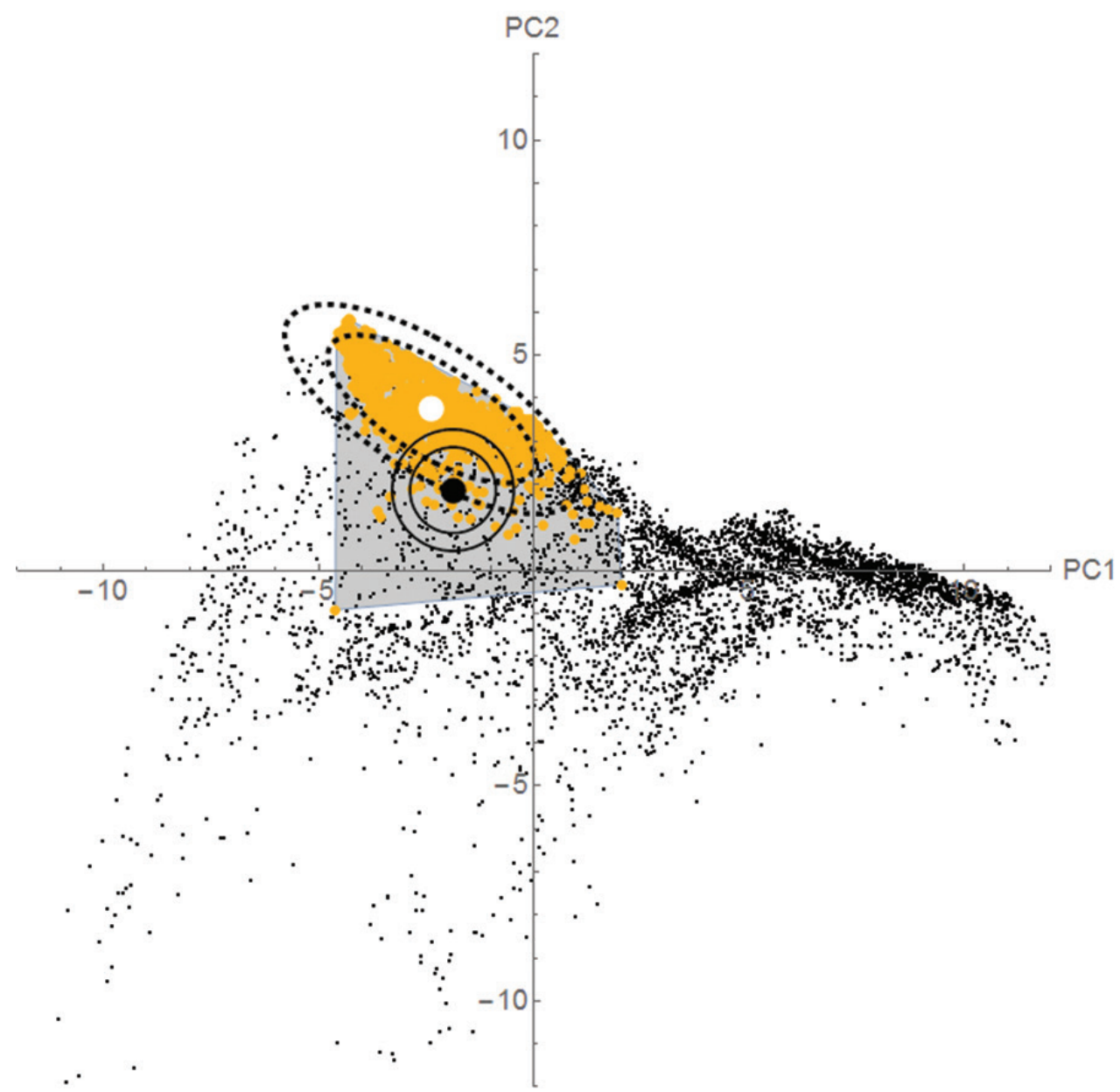

Figure 2. GBIF points for Dipodomys merriami in environmental space, showing differences between methods for delimiting niches and calculating niche-centroid distances. The black circle is the centroid of the convex hull (gray-shaded polygon), showing the strong effect of one outlier point. The white circle is the centroid of a $95 \%$ minimum volume ellipsoid that is able to ignore the outlier. Circles are Euclidean distances of radii 1 and 2, for the convex hull centroid; the dashed ellipsoids are the equivalent distances (Mahalanobis distances) taking into account the covariance shown by the points in gold (see text). Note the striking differences between the two methodologies in both shape of the niche estimated and the distances that result. In particular, note that the centroid estimated via convex hulls falls at the periphery of the cloud of points for the species' occurrence.

niche: a $\mathrm{CH}$ and a minimum volume ellipsoid (MVE). The centroids of both objects (around the same US data) are located in very different positions in niche space.

4) The data provided in the Supplementary Materials of Dallas et al. (2017) reveals that the population-density data points have coordinates with precision of $10^{-3}-10^{-4}$, a geo-referencing precision of $100 \mathrm{~m}$ or finer. However, the climate data used in the paper have a resolution of $-0.042^{\circ}$, or squares of $-4600 \mathrm{~m}$ on a side. This 
means that multiple abundance data points may fall within a single climate pixel, introducing a further problem in the analysis of Dallas et al. (2017), as shown in lines 365-370 of the code provided by the authors. Correcting this methodological problem leaves 40 instead of 81 species for mammals and 49 instead of 63 species for fishes with $>10$ different abundance/climate points; all of the bird species and 165 of the 166 tree species had $>10$ points.

\section{Conclusions}

Dallas et al. (2017) provide the largest-scale analysis available to date of relationships between population density and positions in geographic and environmental spaces. Their negative results contrast with previous empirical work (Yańez-Arenas et al. 2012, Martínez-Meyer et al. 2013) and with theoretical arguments supporting such a relationship (Maguire 1973, Osorio-Olvera et al. 2016).

In this communication, we identify a series of methodological problems underlying the results of Dallas et al. (2017). Of course, in practice, a variety of factors determine population densities, not necessarily the same factors identified by niche modeling (Mi et al. 2017). Among these, many important ones are anthropogenic. These are ignored by Dallas et al. (2017) and by ourselves because we focus on the theoretical possibility that the internal structure of the niche contributes to population density. We suggest that this important question remains far from settled.

\section{Author contribution}

JS and TP discussed and conceived the paper. JS wrote the first version and TP rewrote the second version. LO reviewed the code in Dallas et al. (2017), found the imprecisions we reported and discussed the ideas with JS and TP. JS reanalyzed a whole section of the data in Dallas et al. (2017). TP worked on the D. merriamii example.

\begin{tabular}{l|l|l}
\hline Authors & Contribution & $\mathrm{ACl}$ \\
\hline JS & 0.4 & 1.333 \\
\hline TP & 0.3 & 0.857 \\
\hline LO & 0.3 & 0.857 \\
\hline
\end{tabular}

\section{References}

Dallas T, Decker RR, Hastings A (2017) Species are not most abundant in the centre of their geographic range or climatic niche. Ecology Letters 20: 1526-1533. https://doi. org/10.1111/ele.12860 
Maguire B (1973) Niche response structure and the analytical potentials of its relationship to the habitat. American Naturalist 107: 213-246. https://doi.org/10.1086/282827

Martínez-Meyer E, Díaz-Porras D, Peterson AT, Yáñez-Arenas C (2013) Ecological niche structure and rangewide abundance patterns of species. Biology Letters 9: 20120637. https:// doi.org/10.1098/rsbl.2012.0637

Mi C, Huettmann F, Sun R, Guo Y (2017) Combining occurrence and abundance distribution models for the conservation of the Great Bustard. PeerJ 5: e4160. https://doi.org/10.7717/ peerj. 4160

Osorio-Olvera L, Falconi M, Soberón J (2016) Sobre la relación entre idoneidad del hábitat y la abundancia poblacional bajo diferentes escenarios de dispersión. Revista Mexicana de Biodiversidad 87: 1080-1088. https://doi.org/10.1016/j.rmb.2016.07.001

Patterson BD, Ceballos G, Sechrest W, Tognelli MF, Brooks T, Luna L, Ortega P, Salazar I, Young BE (2003) Digital distribution maps of the mammals of the Western Hemisphere. CD-ROM compiled by NatureServe.

Sagarin R (2002) The 'abundant centre' distribution: to what extent is it a biogeographical rule? Ecology Letters 5: 137-147. https://doi.org/10.1046/j.1461-0248.2002.00297.x

Sullivan BL, Wood CL, Iliff MJ, Bonney RE, Fink D, Kelling S (2009) eBird: A citizen-based bird observation network in the biological sciences. Biological Conservation 142: 22822292. https://doi.org/10.1016/j.biocon.2009.05.006

Syväranta J, Lensu A, Marjomäki TJ, Oksanen S, Jones RI (2013) An empirical evaluation of the utility of convex hull and standard ellipse areas for assessing population niche widths from stable isotope data. PLoS ONE 8: e56094. https://doi.org/10.1371/journal.pone.0056094

Van Aelst S, Rousseeuw P (2009) Minimum volume ellipsoid. Wiley Interdisciplinary Reviews: Computational Statistics 1: 71-82. https://doi.org/10.1002/wics.19

Yañez-Arenas C, Martínez-Meyer E, Mandujano S, Rojas-Soto O (2012) Modelling geographic patterns of population density of the white-tailed deer in central Mexico by implementing ecological niche theory. Oikos 121: 2081-2089. https://doi.org/10.1111/j.16000706.2012.20350.x 